\title{
Interaction of Carmustine Tautomers with Adenine - DFT Study
}

\section{Lemi Türker}

Department of Chemistry, Middle East Technical University, Üniversiteler, Eskişehir Yolu No: 1, 06800 Çankaya/Ankara, Turkey; e-mail: 1turker@gmail.com; lturker@metu.edu.tr

\section{Abstract}

Carmustine is a chemotherapic substance used in treatment of various cancers. In the present study, within the constraints of density functional theory (B3LYP/6-31++G(d,p)), tautomerism of carmustine has been investigated. It may undergo 1,3-type proton tautomerism, however the obtained data for vacuum conditions indicated that the equilibrium concentration of the enol type tautomer should be low. Afterwards, interactions of those tautomers with adenine, a constituent base of DNA and RNA, have been investigated. The composites (1:1) are electronically stable. Their heat of formations are exothermic and the free energy of formation values are favorable. Some of their calculated properties (structural, physicochemical and quantum chemical) are obtained and discussed.

\section{Introduction}

Carmustine (BCNU, BiCNU) chemically is bis-chloroethylnitrosourea. A more systematic name for is 1,3-bis(2-chloroethyl)-1-nitrosourea. It is used as an anti-cancer (antineoplastic or cytotoxic) chemotherapy drug. Carmustine requires activation in the liver to form its active intermediates which act by modifying and cross linking purine bases in DNA. This medication is classified as an "alkylating agent". Carmustine acts on DNA during all phases of the cell cycle, thus resulting in disruption of DNA function, causing cell cycle arrest, and apoptosis. This agent also carbamoylates proteins, including DNA repair enzymes, resulting in an enhanced cytotoxic effect [1-5]. Carmustine is highly lipophilic and crosses the blood-brain barrier readily. It is a drug

Received: September 30, 2020; Accepted: November 9, 2020

Keywords and phrases: carmustine, BCNU, BiCNU, bis-chloroethylnitrosourea, tautomerism, adenine, DFT. Copyright (C) 2021 Lemi Türker. This is an open access article distributed under the Creative Commons Attribution License, which permits unrestricted use, distribution, and reproduction in any medium, provided the original work is properly cited. 
which is mainly used for the treatment of brain tumors, multiple myeloma, Hodgkin's disease and non-Hodgkin's lymphomas. Carmustine exists as a solid, slightly soluble in water, and a very weakly acidic compound (based on its pKa). Carmustine has been detected in multiple biofluids, such as urine and blood. Within the cell, carmustine is primarily located in the cytoplasm. Outside of the human body, carmustine can be found in mollusks. This makes carmustine a potential biomarker for the consumption of this food product. Carmustine is formally rated as a probable carcinogen and is also a potentially toxic compound [6]. Though, carmustine is highly effective drug used in chemotherapy, its merits and demerits go simultaneously. There are some adverse effects associated with it while treatment of the patients including hepatotoxicity. Myelosuppression and pulmonary toxicity are the other ones. The toxicity of a drug is totally dependent upon dosage manner, so is with Carmustine $[6,7]$. In clinical trials, carmustine has been shown to cross the blood-brain barrier and be effective against previously untreated central nervous system leukemia. Its dose-limiting factor is delayed hematopoietic toxicity.

In the present study, firstly tautomerism in carmustine and then interaction of those tautomers with adenine, which is a nucleic acid base, have been considered within the realm of density functional theory (DFT).

\section{Method of Calculations}

In the present study, the initial structural optimizations of all the structures leading to energy minima have been achieved by using MM2 method followed by semi-empirical PM3 self-consistent fields molecular orbital (SCF MO) method [8, 9] at the restricted level $[10,11]$. Subsequent optimizations were achieved at Hartree-Fock level using various basis sets. Then, the structural optimizations were managed within the framework of density functional theory (DFT) [12, 13] at the level of B3LYP/6$31++\mathrm{G}(\mathrm{d}, \mathrm{p})[11,14]$. The exchange term of B3LYP consists of hybrid Hartree-Fock and local spin density (LSD) exchange functions with Becke's gradient correlation to LSD exchange $[13,15]$. The correlation term of B3LYP consists of the Vosko, Wilk, Nusair (VWN3) local correlation functional [16] and Lee, Yang, Parr (LYP) correlation correction functional [17]. Also, the vibrational analyses have been done. The total electronic energies are corrected for the zero point vibrational energy (ZPE). The normal mode analysis for each structure yielded no imaginary frequencies for the $3 N-6$ vibrational degrees of freedom, where $N$ is the number of atoms in the system. This 
indicates that the structure of each molecule corresponds to at least a local minimum on the potential energy surface. All these calculations were done by using the Spartan 06 package program [18].

\section{Results and Discussion}

Since tautomers having different structures possess dual reactivity, it is anticipated that carmustine exhibits 1,3-proton tautomerism and should display variable biological properties (beside others) depending on its tautomer content (allelotropic mixture [19, 20]). Note that substances which are isomeric under certain conditions are tautomeric under more drastic conditions [19, 20].

\subsection{Tautomers}

Carmustine possesses $\mathrm{N}-\mathrm{H}$ moiety linked to the carbonyl group which may undergo 1,3-type proton tautomerism.<smiles>[R]NC(=O)N([R])[N+](=O)[O-]</smiles><smiles>[R]N=C(O)N([R])[O-]</smiles>

R: $\mathrm{CH}_{2} \mathrm{CH}_{2} \mathrm{Cl}$

Figure 1 shows the two tautomeric structures of carmustine, the amide and iminol forms. It also displays the direction of the dipole moment vector in each case. In the amide form the $\mathrm{NH}$ moiety is next to the nitroso group. The distance between them is just $1.86 \AA$.
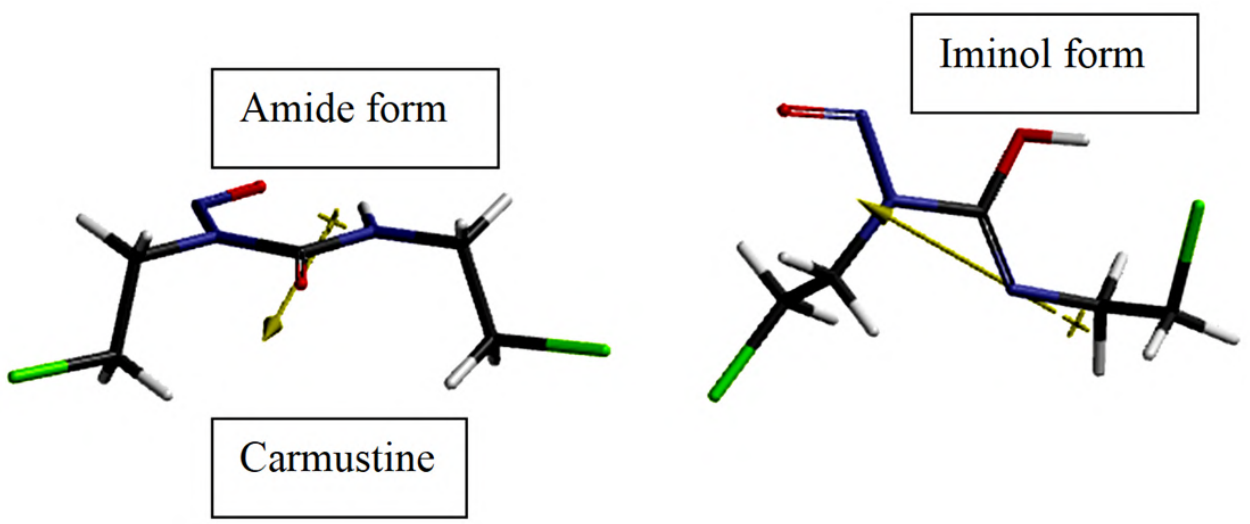

Figure 1. Optimized structures of the tautomers. 
Figure 2 shows the calculated bond lengths of the tautomers considered. The data reveals that two $\mathrm{N}(\mathrm{C}=\mathrm{O})$ bond lengths in the amide form are quite different indicating that some electron population unsymmetrically spread over the carbonyl group.
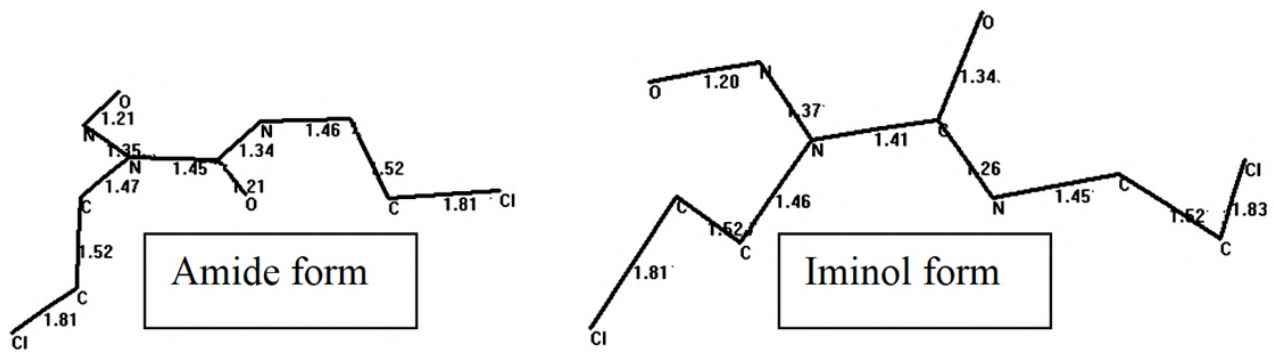

Figure 2. Calculated bond lengths of the tautomers (Hydrogens omitted).

Figure 3 shows the electrostatic potential (ESP) charges developed on the atoms of the optimized structures. Note that the ESP charges are obtained by the program based on a numerical method that generates charges that reproduce the electrostatic potential field from the entire wavefunction [18]. Also note the change of the direction of the dipole moment vectors.
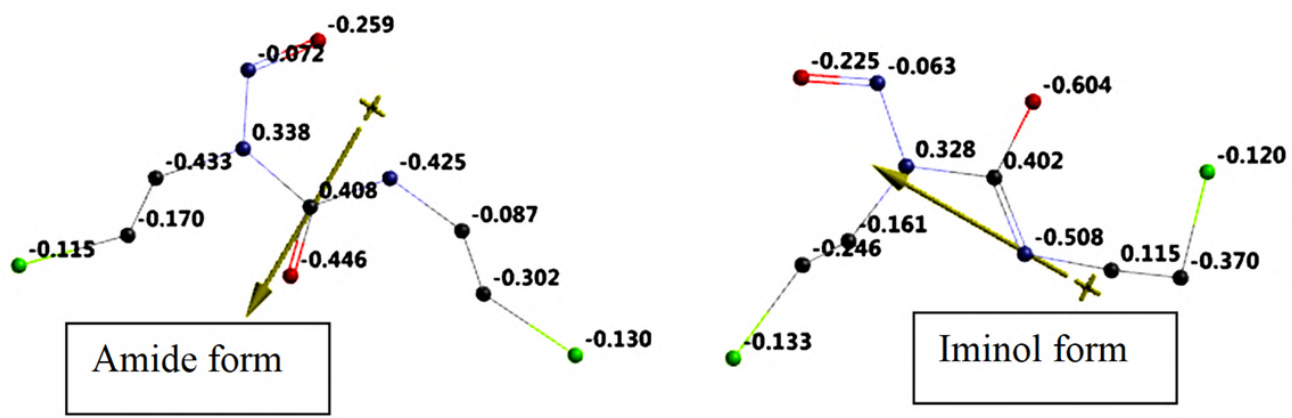

Figure 3. Electrostatic potential charges on atoms of the tautomers (Hydrogens omitted).

Figure 4 stands for the electrostatic potential maps of the tautomers considered. In the figure red/reddish and blue/ green regions stand for negative and positive potential fields, respectively. As seen in the figure, the negative potential field located over the carbonyl moiety of the amide form shifts to nearby of the nitroso moiety in the iminol form. Also an intensely positive potential region appears where the dipole moment vector originates. 

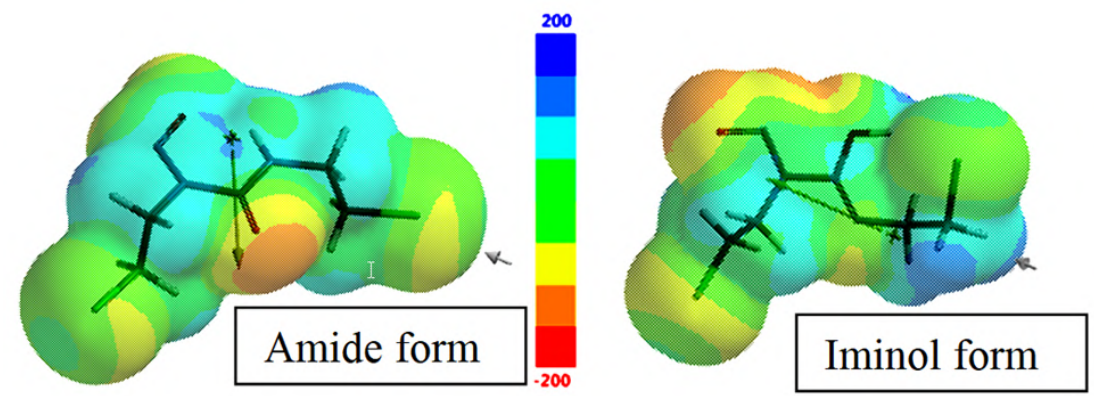

Figure 4. Electrostatic potential maps of the tautomers considered.

Table 1 shows some of the properties of the tautomers. Although, the ovality and polarizability values are quite comparable, the dipole moment and $\log \mathrm{P}$ values are somewhat different for the amide and iminol forms. In the table PSA stands for polar surface area.

Table 1. Some properties of the tautomers considered.

\begin{tabular}{lccccc}
\hline Structure & $\begin{array}{c}\text { Dipole } \\
\text { moment }\end{array}$ & Ovality & Polarizability & $\log \mathbf{P}$ & $\operatorname{PSA}\left(\AA^{2}\right)$ \\
\hline Amide form & 1.65 & 1.40 & 54.57 & 1.11 & 51.763 \\
Iminol form & 4.99 & 1.39 & 54.55 & 1.99 & 53.165 \\
\hline
\end{tabular}

Dipole moment in debye unit.

The higher dipole moment of the iminol form might increase the equilibrium concentration of it in polar solvents, depending on the solvent polarity. For instance, 2hydroxypyridine/ 2-pyridone tautomerism favors the pyridone form because it has much higher dipole moment due to its charge separated mesomeric form [21].

Table 2 lists some of the energies of the structures considered where E, ZPE and $\mathrm{E}_{\mathrm{C}}$ stand for the total electronic energy, zero point vibrational energy and the corrected total electronic energy, respectively. The tautomers are electronically stable, however the data reveal that the iminol form is electronically less stable as compared to the amide form.

Table 2. Some energies of the tautomers considered.

\begin{tabular}{lccc}
\hline Structure & $\mathbf{E}$ & ZPE & $\mathbf{E}_{\mathbf{C}}$ \\
\hline Amide form & -3757635.57 & 410.03 & -3757225.54 \\
Iminol form & -3757552.76 & 408.56 & -3757144.2 \\
\hline
\end{tabular}

Energies in $\mathrm{kJ} / \mathrm{mol}$. 
Table 3 shows some thermodynamic properties of the tautomers. They have exothermic heat of formation values. The amide form is more exothermic and more favorable Gibbs' free energy of formation possessor.

The tautomeric conversion of the amide to iminol form is characterized with $\Delta \mathrm{G}_{\text {reaction }}^{\circ}=\mathrm{G}_{\text {Iminol }}^{\circ}-\mathrm{G}_{\text {Amide }}^{\circ}$ which has the numerical value of 0.003126 au. The equilibrium constant $\mathrm{K}_{\text {eq }}$ value is given by $\mathrm{K}_{\mathrm{eq}}=\mathrm{e}^{-1060 \Delta \text { Greaction }}$ at $298^{\circ} \mathrm{K}$ [22]. The numerical value is 0.036386 . So, carmustine mostly prefers the amide form. The underlying reason could be the existence of some extent of hydrogen bonding between the $\mathrm{N}-\mathrm{H}$ and nitroso oxygen atom because as seen in the optimized structure (see Figure 1) of carmustine they are next to each other, so that the hydrogen atom is somewhat fixed.

Table 3. Some thermodynamic properties of the tautomers considered.

\begin{tabular}{lcccc}
\hline Structure & $\mathbf{H}^{\mathrm{o}}(\mathbf{a u})$ & $\mathbf{S}^{\mathrm{o}}\left(\mathbf{J} / \mathbf{m o l}^{\mathrm{o}}\right)$ & $\mathbf{G}^{\mathrm{o}}(\mathbf{a u})$ & $\mathbf{C v}\left(\mathbf{J} / \mathbf{m o l}^{\mathbf{o}}\right)$ \\
\hline Amide form & -1431.03858 & 437.35 & -1431.08824 & 136.49 \\
Iminol form & -1431.00759 & 434.95 & -1431.05698 & 138.25 \\
\hline
\end{tabular}

Figure 5 shows some of the molecular orbital energy levels of the tautomers. Note the smaller energy gap between the LUMO and NEXTLUMO levels in the iminol form as compared to the amide case.
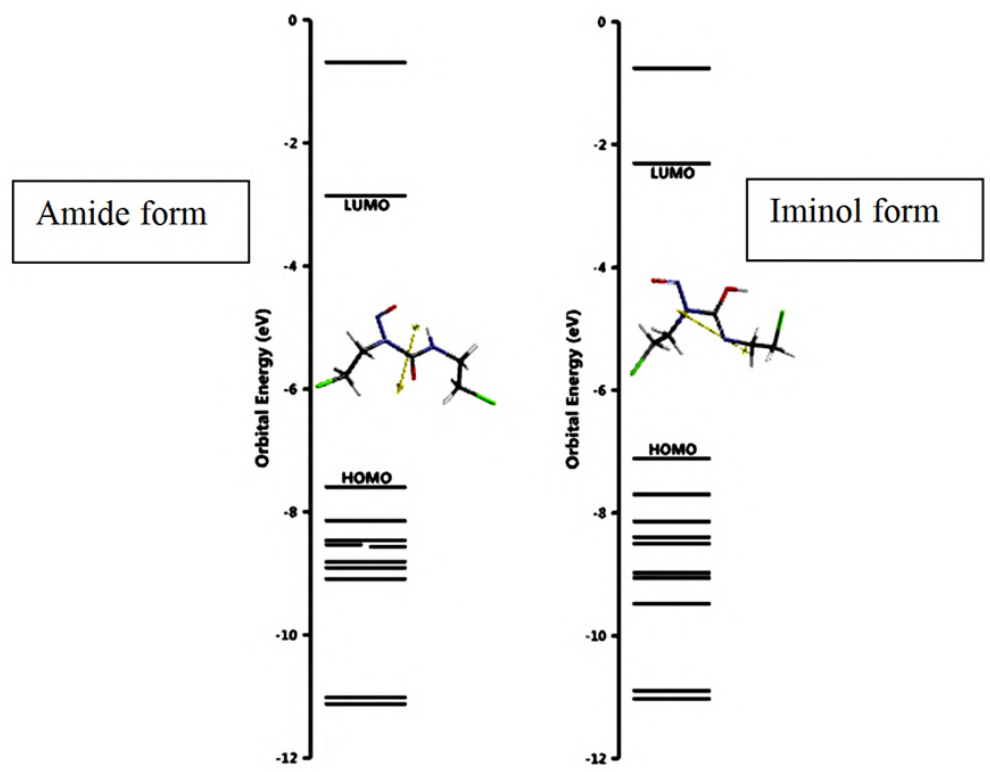

Figure 5. Some molecular orbital energies of the tautomers considered. 
Table 4 lists the HOMO, LUMO energies and the interfrontier molecular orbital energy gap $\left(\varepsilon_{\text {LUMO }}-\varepsilon_{\text {Hомо }}\right)$ values $(\Delta \varepsilon)$ for the tautomers. The amide form has lower HOMO and LUMO energies and smaller $\Delta \varepsilon$ value compared to the iminol form.

Table 4. The HOMO, LUMO energies and $\Delta \varepsilon$ values of the tautomers considered.

\begin{tabular}{lccc}
\hline Structure & HOMO & LUMO & $\Delta \boldsymbol{\varepsilon}$ \\
\hline Amide form & -732.67 & -275.62 & 457.05 \\
Iminol form & -686.24 & -222.70 & 463.54 \\
\hline
\end{tabular}

Energies in $\mathrm{kJ} / \mathrm{mol}$.

Figure 6 shows the local ionization potential maps of the tautomers considered. In a local ionization potential map conventionally red regions on the density surface indicate areas from which electron removal is relatively easy, meaning that they are subject to electrophilic attack. On the other hand, regions having blue color represent areas where ionization is relatively difficult.

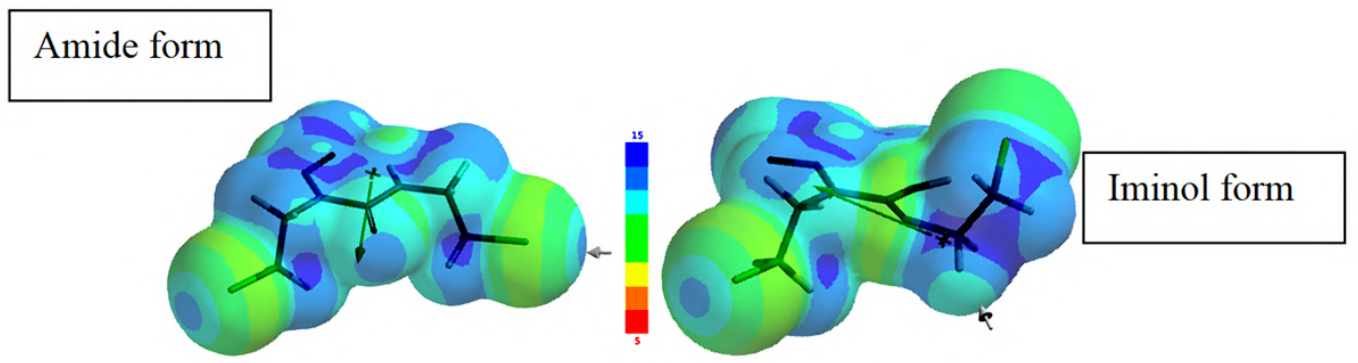

Figure 6. The local ionization potential maps of the tautomers considered.

Figure 7 shows the LUMO maps of the tautomers considered. A LUMO map
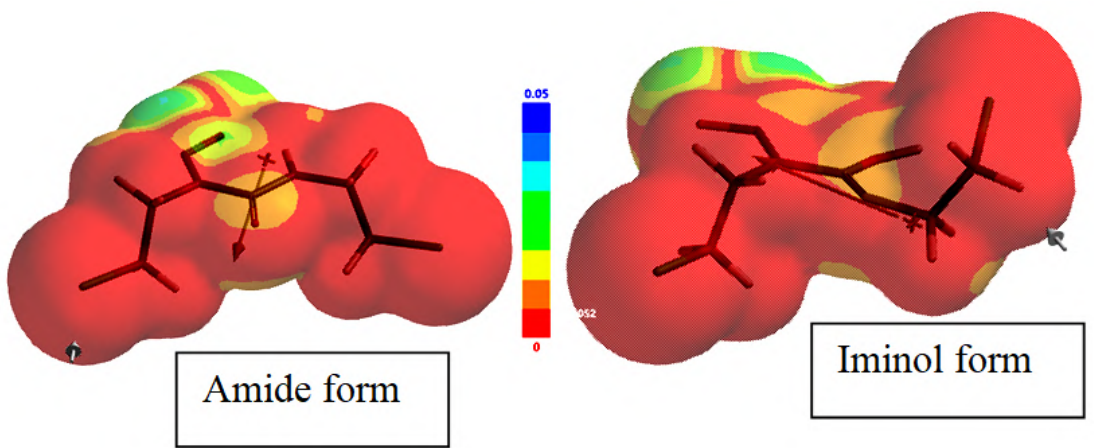

Figure 7. The LUMO maps of the structures considered. 
displays the absolute value of the LUMO on the electron density surface. The blue color stands for the maximum value of the LUMO and the color red, the minimum value. Hence, a nucleophile attacks on the atom having the blue/bluish color.

\subsection{Composites}

Adenine, a purine derivative, is one of the nucleic acid bases involved in the structures of DNA and RNA molecules [23].
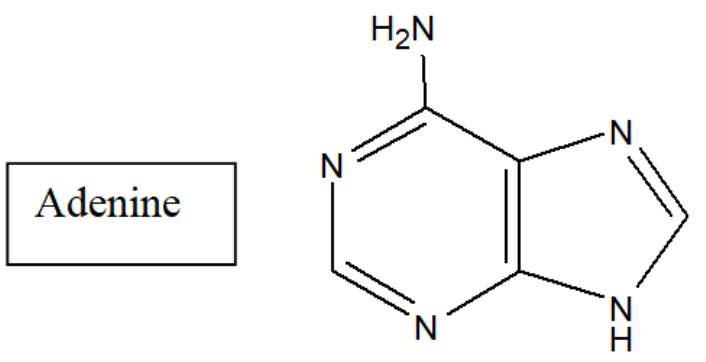

Figure 8 shows the optimized structures of composites (1:1) of carmustine tautomers presently considered with adenine molecule. Note the orientation of the components and the direction of the dipole moment vectors. The adenine molecule orients itself in such a way that the location of $\mathrm{NH}_{2}$ group of adenine in space in each composite seems very different.
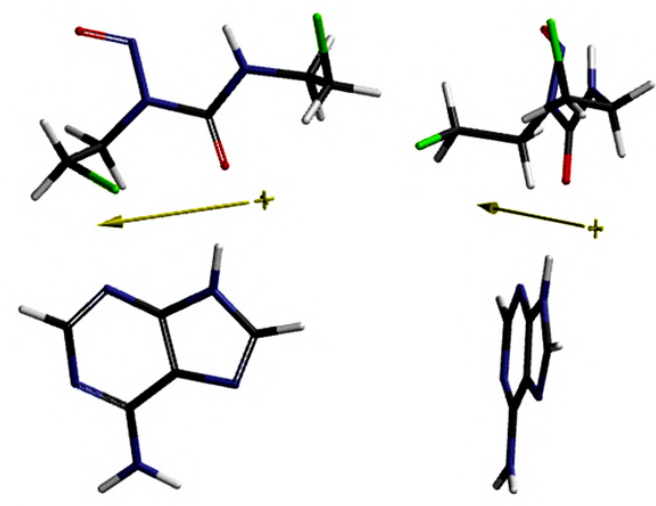

Amide form+Adenine

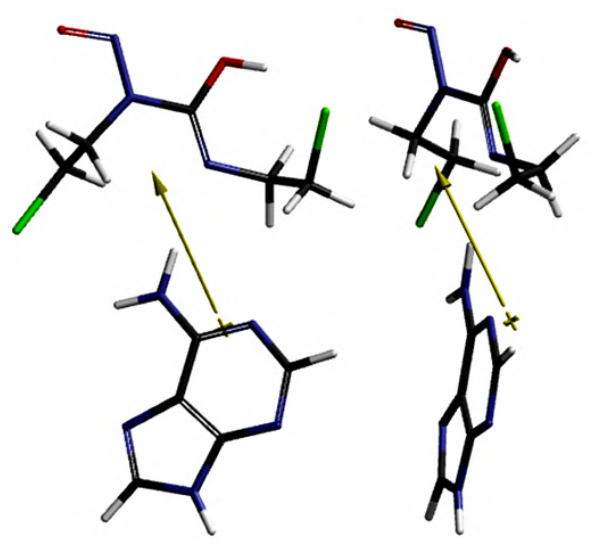

Iminol form+Adenine

Figure 8. Optimized structures of the composites considered (in two different views). 
In the case of composite having the amide form of carmustine, probably some extent of hydrogen bonding occurs between the carbonyl oxygen atom of carmustine and the hydrogen belonging to imidazole ring of the adenine molecule. The distance is about $1.90 \AA$. In the case of the composite with iminol form, the adenine $\mathrm{NH}_{2}$ group is next to the iminol system. Although, the tautomers have $\mathrm{C}-\mathrm{Cl}$ bonds which might be thought to undergo $\mathrm{SN}_{2}$ type reactions with nucleophilic sites of adenine molecule, the optimized structures of the composites do not imply any inclination of it. It might be because of the steric and electronic reasonings.

Table 5 shows some properties of the composites. Note the higher dipole moment value of the iminol composite and the change of sign of the $\log \mathrm{P}$ values in between the composites.

Table 5. Some properties of the composites considered.

\begin{tabular}{lccccc}
\hline Structure & Dipole moment & Ovality & Polarizability & $\log$ P & PSA \\
\hline Amide form+Adenine & 2.89583976 & 1.62 & 64.77 & -0.34 & 106.065 \\
Iminol form+Adenine & 4.36879833 & 1.61 & 64.66 & 0.54 & 107.255 \\
\hline
\end{tabular}

Dipole moment in debye unit. PSA in $\AA^{2}$.

Figure 9 displays the electrostatic potential charges on atoms of the composites.
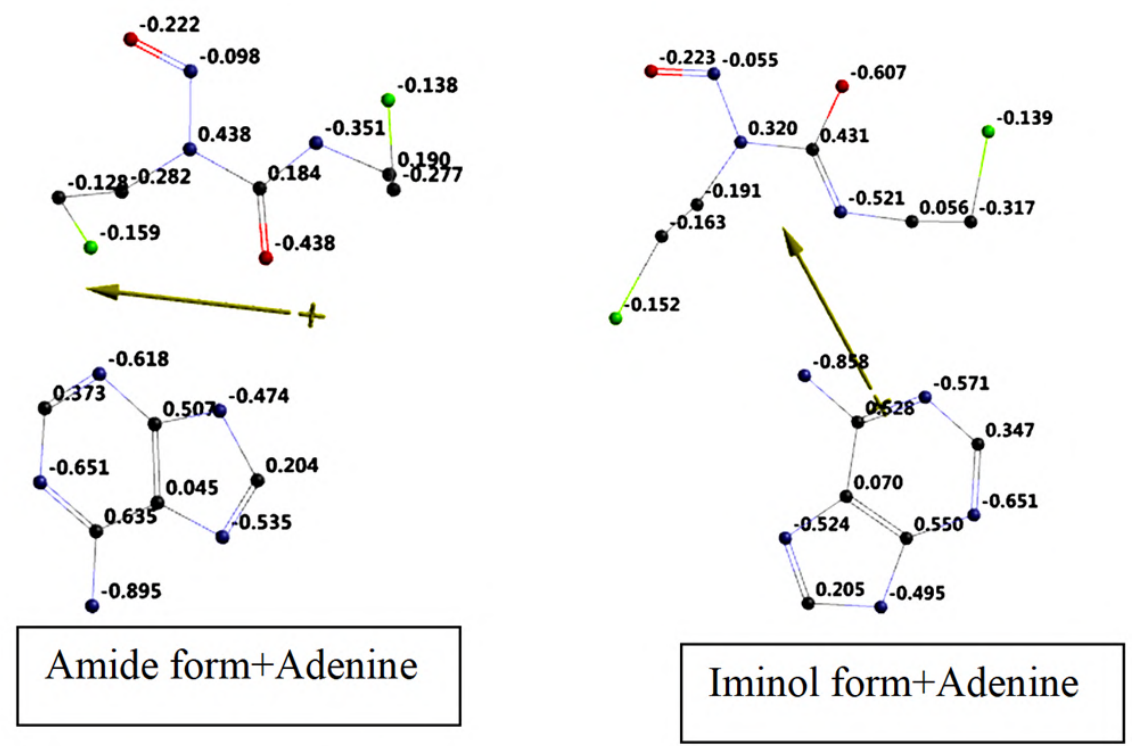

Figure 9. Electrostatic charges on atoms of the composites (Hydrogens omitted). 
Figure 10 shows the electrostatic potential maps of the composites considered. As seen in the figure distribution of the negative and positive potential fields is so that the dipole moment vector in the iminol composite originates from adenine component and points to the iminol component.

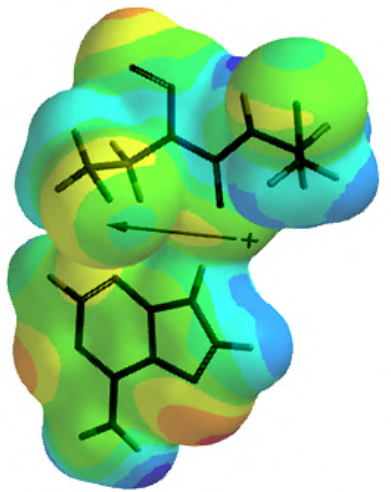

Amide form + Adenine

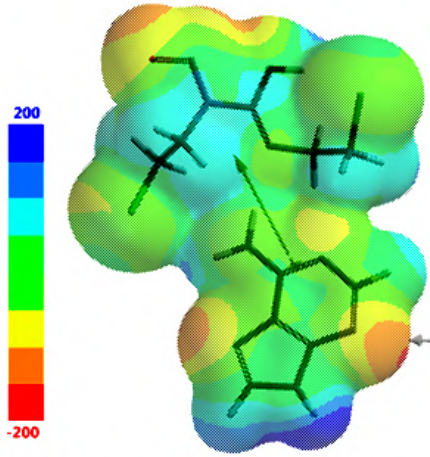

Amide form + Adenine

Figure 10. Electrostatic potential maps of the composites considered.

Table 6 lists some energies of the composites. As seen in the table the carmustine (amide) composite is electronically more stable in the amide form than the iminol case.

Table 6. Some energies of the composites considered.

\begin{tabular}{lccc}
\hline Structure & $\mathbf{E}$ & $\mathbf{Z P E}$ & $\mathbf{E}_{\mathbf{C}}$ \\
\hline Amide form +Adenine & -4984258.98 & 709.35 & -4983549.63 \\
Iminol form+Adenine & -4984153.79 & 707.505 & -4983446.28 \\
\hline
\end{tabular}

Energies in $\mathrm{kJ} / \mathrm{mol}$.

Table 7 contains some thermodynamic properties of the composites considered. Both of the composites possess exothermic heat of formation values such that the amide composite is more exothermic. As for the standard energy of formation values $\left(\mathrm{G}^{\circ}\right)$, the amide composite is more favorable. However, $\Delta \mathrm{G}_{\text {reaction }}^{\circ}$ values are 0.18444 and 0.19282 $\mathrm{au}$, respectively for the composites derived from the amide and iminol tautomers. This occurrence is most probably due to the fact that the composite formations from the starting components are entropically disfavored. 
Table 7. Some thermodynamic properties of the composites considered.

\begin{tabular}{lcccc}
\hline Structure & $\mathbf{H}^{\mathbf{o}}(\mathbf{a u})$ & $\mathbf{S}^{\mathbf{0}}\left(\mathbf{J} / \mathbf{m o l}^{\mathbf{0}}\right)$ & $\mathbf{G}^{\mathbf{o}}(\mathbf{a u})$ & $\mathbf{C v}\left(\mathbf{J} / \mathbf{m o l}^{\mathbf{o}}\right)$ \\
\hline Amide form+Adenine & -1898.11364 & 559.26 & -1898.17715 & 236.41 \\
Iminol form+Adenine & -1898.07431 & 556.67 & -1898.13753 & 237.96 \\
\hline
\end{tabular}

Figure 11 displays some molecular orbital levels of the composites considered. The iminol composite has larger frontier molecular orbital energy gap but narrower LUMONEXTLUMO gap compared to the respective values of the amide composite.

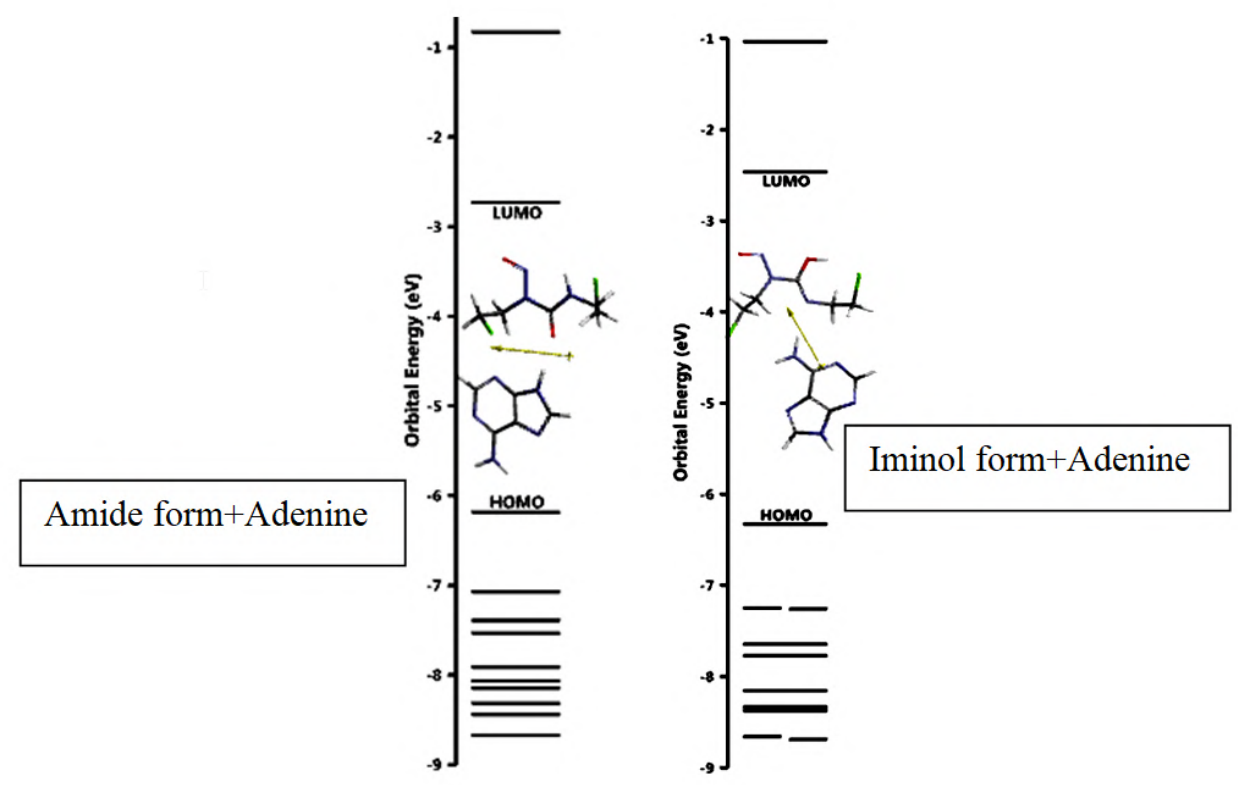

Figure 11. Some molecular orbital energy levels of the composites considered.

Table 8 includes the HOMO, LUMO energies and $\Delta \varepsilon$ values of the composites. As seen in the table the amide composite has higher HOMO but lower LUMO energy than the iminol composite. Consequently it possesses a narrower interfrontier molecular energy gap $(\Delta \varepsilon)$ than the iminol case.

Table 8. The HOMO, LUMO energies and $\Delta \boldsymbol{\varepsilon}$ values of the composites considered.

\begin{tabular}{lccc}
\hline Structure & HOMO & LUMO & \multicolumn{1}{c}{$\Delta \boldsymbol{\varepsilon}$} \\
\hline Amide form+Adenine & -596.66 & -263.43 & 333.24 \\
Iminol form+Adenine & -610.62 & -237.49 & 373.13 \\
\hline
\end{tabular}

Energies in $\mathrm{kJ} / \mathrm{mol}$. 
Figure 12 shows the calculated UV-VIS spectra (time-dependent DFT, TDDFT) of the species considered. The influence of interaction with adenine molecule is apparent in the spectra of the composites where a shoulder-like absorption peak emerges in both of the relevant spectra. Note that some of the peaks of the composites have been somewhat shifted to visible side as compared to the parent tautomeric species and adenine.
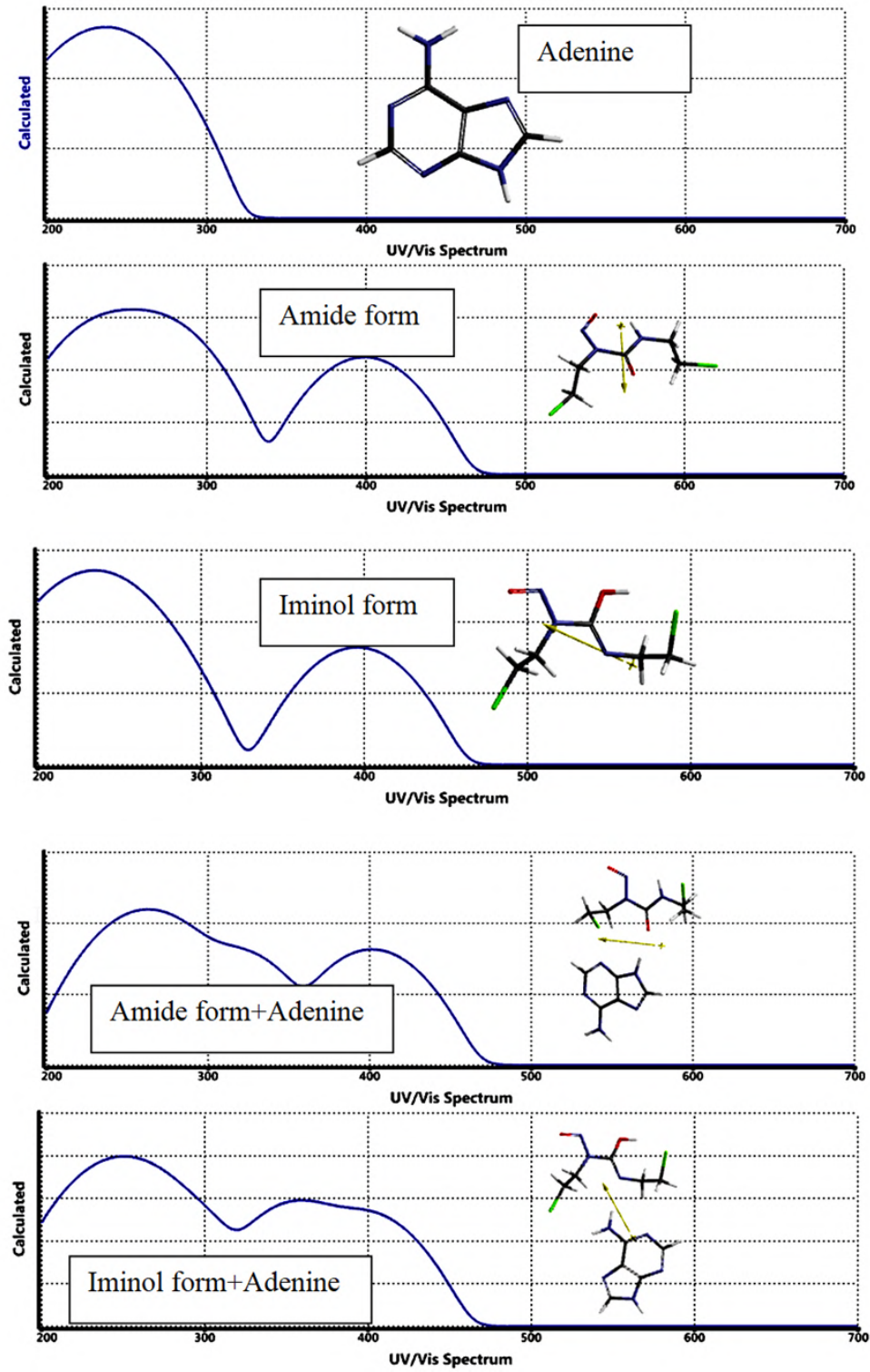

Figure 12 . Calculated UV-VIS spectra of the species considered. 


\section{Conclusion}

The present DFT study at the level of application indicates that in vacuum conditions the concentration of iminol tautomer of carmustine is low. However, its concentration might be increased in polar solvents, because its dipole moment is higher than that of the amide form. Also, interaction with some additives to form composites might increase the equilibrium concentration of the iminol form. However, the effect of hydrogen-bond donor and acceptor solvents should be rather different. The present study indicates that adenine interacts in different extents with the tautomers considered, although the composite formation is not favored based on thermodynamic considerations. However carmustine metabolized in liver might exhibit a different behavior.

\section{References}

[1] J.D. Ahlgren and J.S. Macdonald (Eds.), Gastrointestinal Oncology, Philadelphia: Lippincott Co., 1992.

[2] T.H. Wasserman, M. Slavik and S.K. Carter, Review of CCNU in clinical cancer therapy, Cancer Treatment Reviews 1(2) (1974), 131-151. https://doi.org/10.1016/S0305-7372(74)80015-0

[3] C.G. Moertel, Chemotherapy of gastrointestinal cancer, N Engl J Med. 299 (1978), 10491052. https://doi.org/10.1056/NEJM197811092991906

[4] J.D. Boice, Jr., M.H. Greene, J.Y. Killen, Jr., S.S. Ellenberg, R.J. Keehn, E. McFadden, T.T. Chen, J.F. Fraumeni, Jr., Leukemia and Preleukemia after Adjuvant Treatment of Gastrointestinal Cancer with Semustine (Methyl-CCNU), N Engl J Med. 309 (1983), 1079-1084. https://doi.org/10.1056/NEJM198311033091802

[5] H.C. Bock, M.J.A. Puchner, F. Lohmann, M. Schütze, S. Koll, R. Ketter, R. Buchalla, N. Rainov, S.R. Kantelhardt, V. Rohde and A. Giese, First-line treatment of malignant glioma with carmustine implants followed by concomitant radiochemotherapy: a multicenter experience, Neurosurg. Rev. 33 (2010), 441-449. https://doi.org/10.1007/s10143-010-0280-7

[6] J.K. Aronson (Editor-in-Chief), Nitrosourea cytotoxic drugs, Meyler's Side Effects of Drugs: The International Encyclopedia of Adverse Drug Reactions and Interactions, $16^{\text {th }}$ ed., Amsterdam: Elsevier, 2016. https://doi.org/10.1016/B978-0-444-53717-1.01160-4

[7] A.V. Thatishetty, N. Agresti and C.B. O'Brien, Chemotherapy-Induced Hepatotoxicity, Clinics in Liver Disease 17(4) (2013), 671-686.

https://doi.org/10.1016/j.cld.2013.07.010 
[8] J.J.P. Stewart, Optimization of parameters for semiempirical methods I. Method, $J$. Comput. Chem. 10 (1989), 209-220. https://doi.org/10.1002/jcc.540100208

[9] J.J.P. Stewart, Optimization of parameters for semi empirical methods II. Application, $J$. Comput. Chem. 10 (1989), 221-264. https://doi.org/10.1002/jcc.540100209

[10] A.R. Leach, Molecular Modeling, Essex: Longman, 1997.

[11] P. Fletcher, Practical Methods of Optimization, New York: Wiley, 1990.

[12] W. Kohn and L. Sham, Self-consistent equations including exchange and correlation effects, J. Phys. Rev. 140 (1965), 1133-1138. https://doi.org/10.1103/PhysRev.140.A1133

[13] R.G. Parr and W. Yang, Density Functional Theory of Atoms and Molecules, London: Oxford University Press, 1989.

[14] C.J. Cramer, Essentials of Computational Chemistry, Chichester, West Sussex: Wiley, 2004.

[15] A.D. Becke, Density-functional exchange-energy approximation with correct asymptotic behavior, Phys. Rev. A 38 (1988), 3098-3100. https://doi.org/10.1103/PhysRevA.38.3098

[16] S.H. Vosko, L. Wilk and M. Nusair, Accurate spin-dependent electron liquid correlation energies for local spin density calculations: a critical analysis, Can. J. Phys. 58 (1980), 1200-1211. https://doi.org/10.1139/p80-159

[17] C. Lee, W. Yang and R.G. Parr, Development of the Colle-Salvetti correlation-energy formula into a functional of the electron density, Phys. Rev. B 37 (1988), 785-789. https://doi.org/10.1103/PhysRevB.37.785

[18] SPARTAN 06, Wavefunction Inc., Irvine CA, USA, 2006.

[19] O.Reutov, Theoretical principles of organic chemistry, Moscow: Mir Pub., 1970.

[20] E.V. Anslyn and D.A. Dougherty, Modern Physical Organic Chemistry, Sausalito, California: University Science Books, 2006.

[21] C. Reichardt, Solvents and Solvent Effects in Organic Chemistry, 3rd ed., Weinheim: Wiley-VCH, 2003.

[22] Spartan, Molecular Modeling in Physical Chemistry, Irvine, CA, USA: Wavefunction Inc., 2005.

[23] G. Zubay, Biochemistry, Reading: Addison-Wesley, 1983. 